\title{
Computation of External Aerodynamics for a Canard Rotor/Wing Aircraft
}

\author{
S. A. Pandya and M. J. Aftosmis \\ Mail Stop T-27B \\ NASA Ames Research Center \\ Moffett Field, CA 94035 \\ (pandya,afrosmis)@nas.nasa.gov
}

\begin{abstract}
The aerodynamic loads on a Canard Rotor/Wing vehicle are investigated using inviscid numerical simulations in order to understand the flight characteristics of the vehicle during conversion from rotor craft to fixed-wing flight. A series of numerical simulations at seven azimuthal rotor indices are presented covering a quarter turn of the rotor. With symmetry arguments, these simulations produce 25 data points for a complete rotation. A Cartesian mesh approach is used to compute the flow field about a configuration with faired-over engine inlet and exhaust that matches the wind tunnel geometry. These simulations were performed using meshes with approximately 9Million Cartesian cells. To better understand the aerodynamic effects of the rotor hub on the configuration, the same set of simulations were repeated for a hub-less geometry. Overall loads for both configurations are similar, but are due to somewhat different aerodynamic mechanisms.
\end{abstract}

\section{Introduction}

The Canard Rotor/Wing (CRW) is a vertical takeoff and landing fiight demonstration aircraft built via a joint Boeing/ DARPA effort. Figure 1 shows a 3-view of the configuration. The CRW is similar in concept to the X-wing prototype in that it uses a symmetric rotor with an elliptic airfoil section for takeoff, landing and hover, and that this rotor can be locked in a fixed position for use as a main-wing for highspeed forward flight. During hover, each rotor is cyclically pitched in order to generate lift; while in forward flight, both rotor blades are fixed in the wing position and collectively pitched to generate lift.

During development, analysis and testing indicated that the $\mathrm{X}$-wing design encountered high transient aerodynamic loads during the conversion from rotor craft to fixed-wing flight.

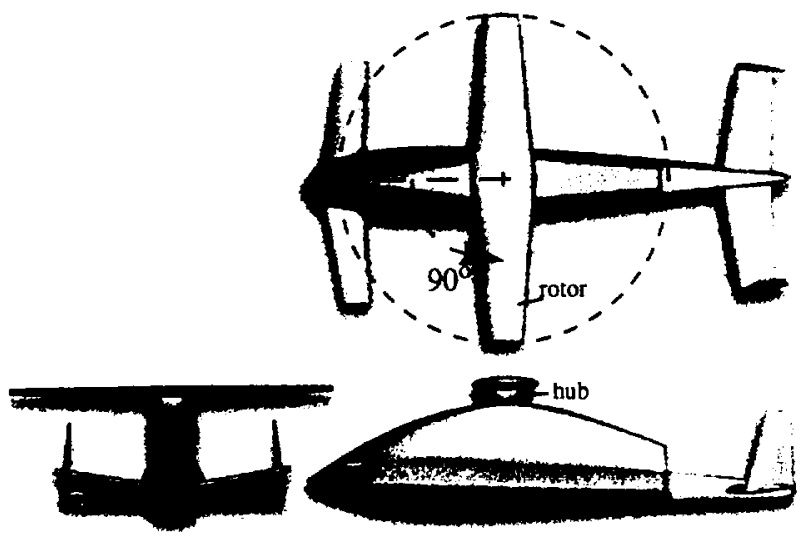

Figure 1: Three-view of the Canard Rotor/Wing flight demonstration aircraft used in the numerical simulations.
The CRW design addresses this issue by generating positive lift on both the canard and horizontal tail during conversion. By deploying the canard flap and the full-flying horizontal tail, enough lift can be produced to maintain altitude, the main rotor can be completely unloaded throughout the conversion maneuver.

Despite the fact that the rotor is unloaded, it still decelerates through a non-uniform free stream, and therefore will still transmit unsteady loads to the airframe. The frequency of the loads will be the harmonics of the rotor speed, but their amplitude is difficult to estimate. Prediction of the amplitude of these unsteady loads is essential in sizing the control surfaces and actuators of the flight control system.

In this work, we use numerical simulations to get quantitative estimates of the magnitude of these unsteady loads and an understanding of the aerodynamic mechanisms which produce them. Work on the X-wing indicated that the most severe loadings occurred during the last few revolutions of the rotor when it turns relatively slowly. Under these conditions, the rotation time-scale is much smaller than that of the flow, making a sequence of steady measurements at fxed rotor azimuthal positions an efficient and viable method to measure and predict the loads. For this reason, the wind tunnel experiments ${ }^{[1]}$ and numerical simulations(in this paper) were carried out with the rotor fixed at a series of azimuthal positions around the rotor disk.

The simulations are performed with the rotor positioned at $0^{\circ}$, $15^{\circ}, 30^{\circ}, 45^{\circ}, 60^{\circ}, 75^{\circ}$, and $90^{\circ}$. Symmetry extends the data from these seven first quadrant rotor positions to fill 25 stations for one $360^{\circ}$ turn of the rotor. The flight conditions for conversion from rotor craft to fixed-wing flight are set at a Mach number of $0.1986(130 \mathrm{Knots})$ and $0^{\circ}$ angle-of-attack. Each of the computations is performed using the Cartesian mesh flow simulation package discussed in references [2-4]. The surface preparation, triangulation, and Cartesian mesh generation capabilities in this package are used to generate the mesh system at each of the azimuthal positions. Two inviscid flow solvers are included in this package and both have been extensively validated in the open literature. ${ }^{[3-5]}$

This paper first presents preliminary solutions obtained on the complete CRW with the hub included. The integrated forces and moments from these simulations are presented as a function of the rotor's azimuthal position. The trends in these forces and moments are explained in terms of the changes in surface pressure. These simulations suggest that the hub has a significant effect on the flow and thus on the vehicle loads. In order to better understand the hemispherical 

hub's contribution to the changes in the flow and the loads, a full series of simulations are presented with the hub removed. Though this case is not realistic in that there is no hardware attaching the rotor to the fuselage, it helps us understand the aerodynamic mechanisms that contribute to the changes in vehicle loads.

\section{Methods}

The process begins with receiving geometry components as CAD solids. The components can be moved, and/or rotated with respect to each other in order to perform parametric studies. In the current case, the rotor is positioned at several azimuthal angles using this feature. Surface triangulations are generated for each of the CAD solids using a combination of CAD tools and auxiliary tools bundled with the Cartesian mesh flow simulation package. These component triangulations are then provided as input into the Cartesian mesh generation system.

After indexing the rotor to the desired azimuthal position, the component triangulations are intersected together to extract the wetted surface of the configuration. The intersection program in the Cartesian package[2] incorporates adaptive precision arithmetic, and includes an automatic tie-breaking routine that consistently resolves geometric degeneracies without user intervention. This tool computes the intersections between closed body triangulations, and retriangulates the intersection neighborhood to join the pieces together into a single triangulation.

The volume mesh generation algorithm takes the intersected surface triangulation as input. This program generates an unstructured Cartesian mesh through subdivision of hexahedral cells of an initially uniform coarse grid. It adapts to the contours of the geometry, thus creating a mesh where the size of the cubic cells is smallest in the neighborhood of the sharpest features of the geometry, while away from the geometry, the grid remains coarse. Specific regions of enhancement can also be defined in order to ensure that a given mesh size is maintained in a specified area. The program also computes the intersections of the geometry with the cells of the mesh which pierce the geometry in order to define polyhedral cut-cells. The faces of these polyhedral cells are then used to specify the tangency boundary condition in the flow solver. Meshes generated by this algorithm at five different rotor indices are presented in Figure 2. The body and cuts from the mesh in the vicinity of the body are shown. Cuts through the mesh, behind and under the body, make up the background of the figures. One small figure shows the canard/flap region in greater detail. The approach in this volume mesh generator preserves the ability to refine the mesh to different degrees in different directions. This makes it possible to avoid generating an excessive number of Cartesian cells in three dimensions.

A Cartesian flow solver that solves the Euler equations is used to compute the flow field. The Euler equations describe the unsteady flow of an inviscid perfect gas and may be expressed in integral form.

$$
\iiint_{\Omega} \frac{\partial}{\partial t} \bar{Q} d V=-\oint_{\partial \Omega}(\bar{F} \cdot \hat{n}) d S
$$

Here $Q$ is the state vector of conserved quantities, and $F$ is the tensor of flux density for an inviscid perfect gas. $\Omega$ refers to an arbitrary control volume and $\partial \Omega$ is its closed boundary with an outward facing surface normal vector $\vec{n}$.

The flow solver is an upwind code that uses an explicit multistage Runge-Kutta time stepping algorithm to drive the solution to a steady state. An optimally damped $2^{\text {nd }}$ order scheme using five Runge-Kutta stages was used to compute the present set of subsonic cases[6]. The flow solver also uses multi-grid convergence acceleration to damp the high frequency error modes. In the present computations, five levels of multi-grid are used. The coarse meshes for the multi-grid computations were generated by a utility in the Cartesian mesh flow simulation package. This utility is documented in reference [3]. Details of this flow solver scheme can be found in the references $[3,4]$.

\section{Simulations}

The solutions are presented below for all rotor settings at a Mach number of 0.1986 and $0^{\circ}$ angle of attack. This corresponds to the conversion scenario where the aircraft is switching from hover to forward flight at 130 Knots. For all simulations, the boundaries of the mesh spanned 20 times the body length in all directions.

The change in the value of lift is used to determine the convergence of the solution. All cases are run untill the value of lift converges to within $1 \%$. Approximately two orders of magnitude drop in the residual on the finest mesh after a full multigrid startup is also required.

\subsection{Simulations with hub}

A simulation of the full geometry with the hub is conducted for seven rotor positions in the first quadrant. As discussed in the introduction, this results in 25 data points, due to symmetry, covering one 360 degree rotation. In this section, we discuss the trends found during these simulations.

Figure 3 shows the body of the CRW, colored by the coefficient of pressure, for all computed rotor positions. In these surface pressure figures, high pressure corresponds to magenta, while suction corresponds to blue. The suction on the top surfaces of the canard and horizontal tail shows that these components are producing lift. This is seen clearly in Figure 5 which shows the componentwise breakdown of the lift. The rotor produces little or no lift in comparison with the canard and horizontal tail. In other words, the aircraft is flying entirely on the lift produced by the canard/flap and the horizontal tail. The aircraft is no longer dependent on the rotor for lift. Figure 5 also shows that the lift produced by the fuselage, the canard, the horizontal tail and the vertical tail does not change very much as the rotor turns. The changes in total lift are almost entirely due to the changes in lift produced by the rotor. Upon close inspection of Figure 3, we realize that most of the surface pressures do not change from rotor position to rotor position. However, the pressure on the 
underside of the rotor seems to change with the changing rotor position as shown in Figure 4 on page 4.
While the changes in pressure are minimal over most of the surface, in the region of the rotor hub fairing the surface pressure changes from rotor position to rotor position. A possible mechanism for changing the flow on the rotor is the down-
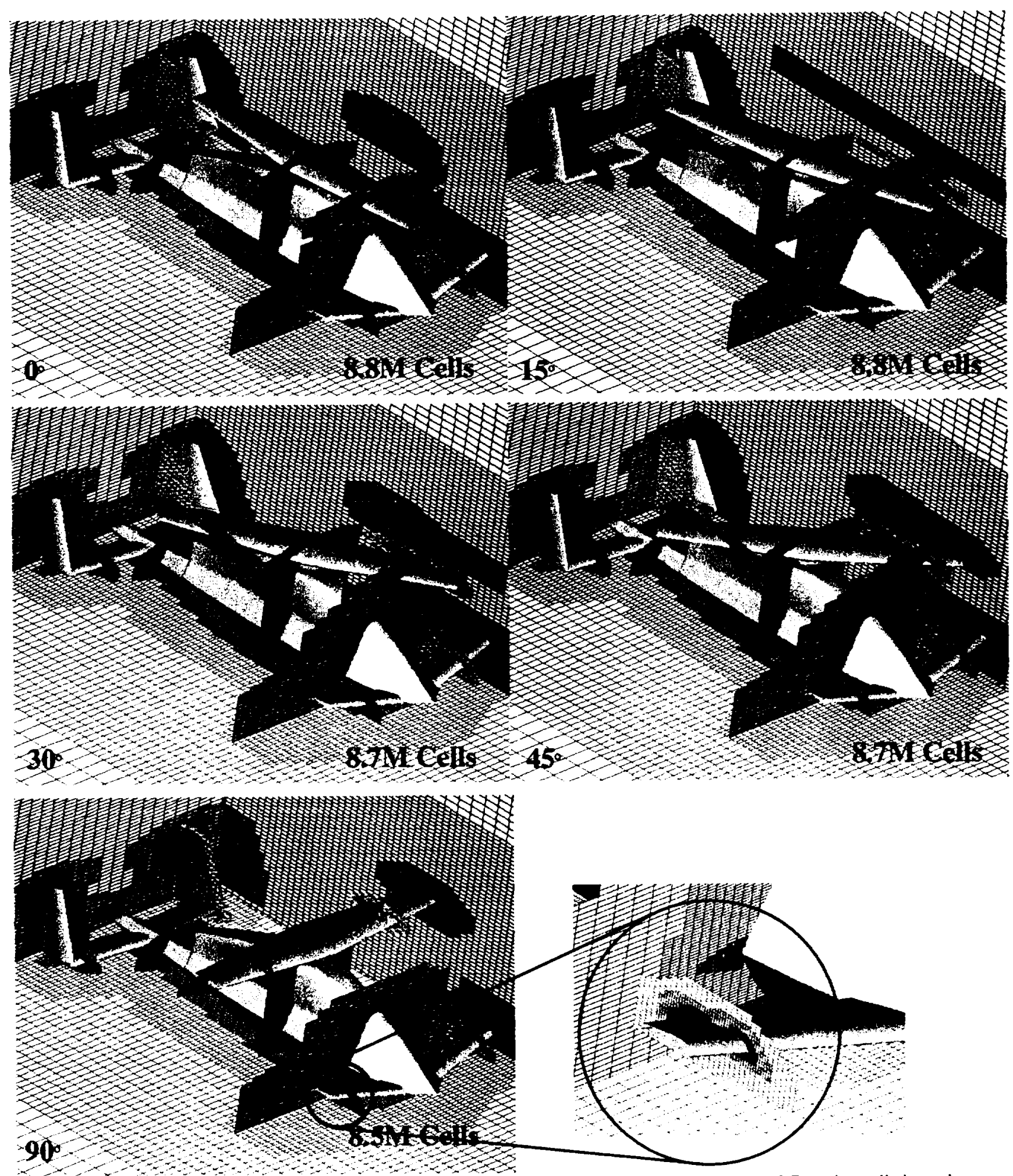

Figure 2: The volume mesh over the CRW at several azimuthal rotor positions. The number of Cartesian cells in each grid is indicated. The close-up shown in the final frame is representative of the resolution in all meshes. 
wash from the canard/flap. If the rotor sees the downwash from the canard/flap, it would produce negative lift simply because the effective angle of attack on the rotor would then be negative. Figure 6 shows streamlines in a vertical plane that cuts the outboard part of the canard/flap for the $90^{\circ}$ rotor position case. This figure shows that while there is a down-

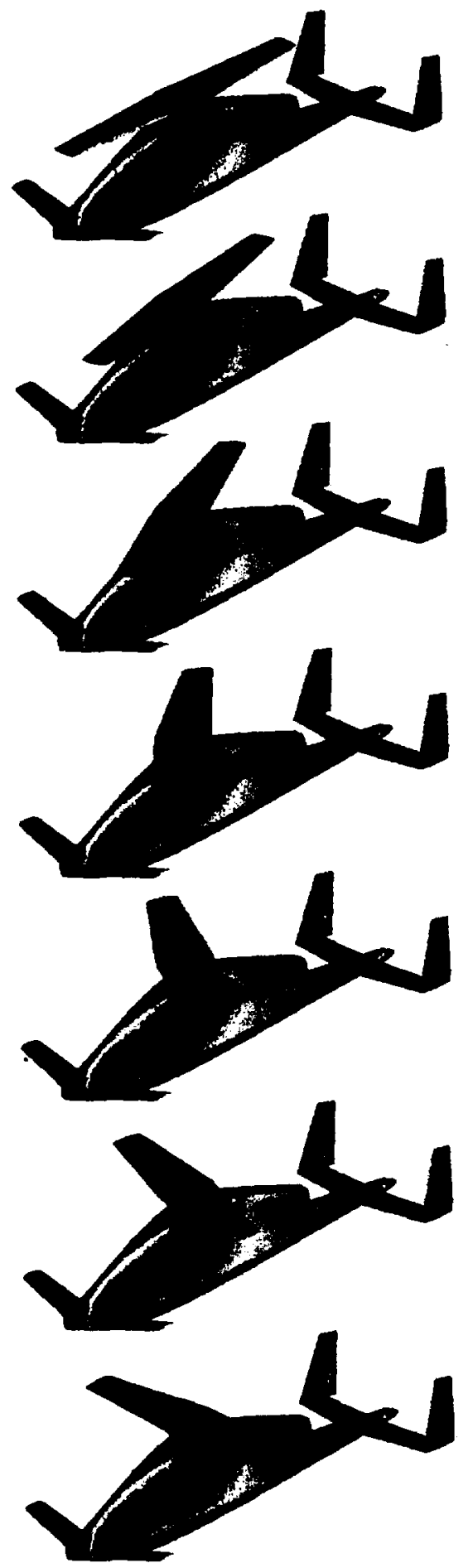

Figure 3: Surface pressure on the CRW for seven rotor positio!

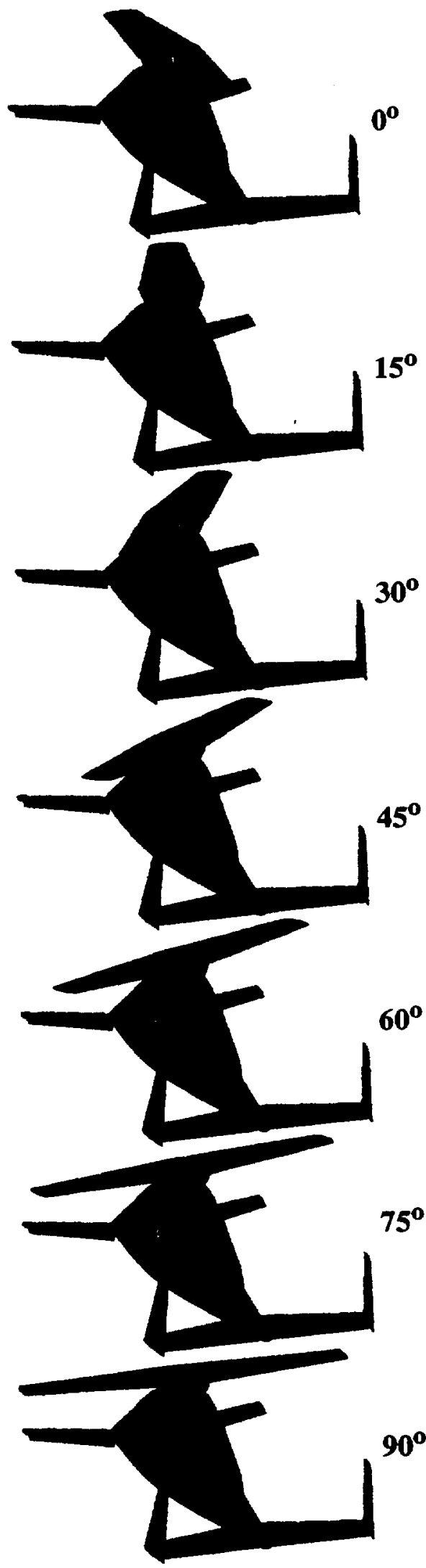

Figure 4: Underview of the surface pressure 

wash associated with the lift generated by the canard/flap, the rotor is not effected by this downwash. Thus, the changes in pressure on the underside of the canard must be due to other effects.

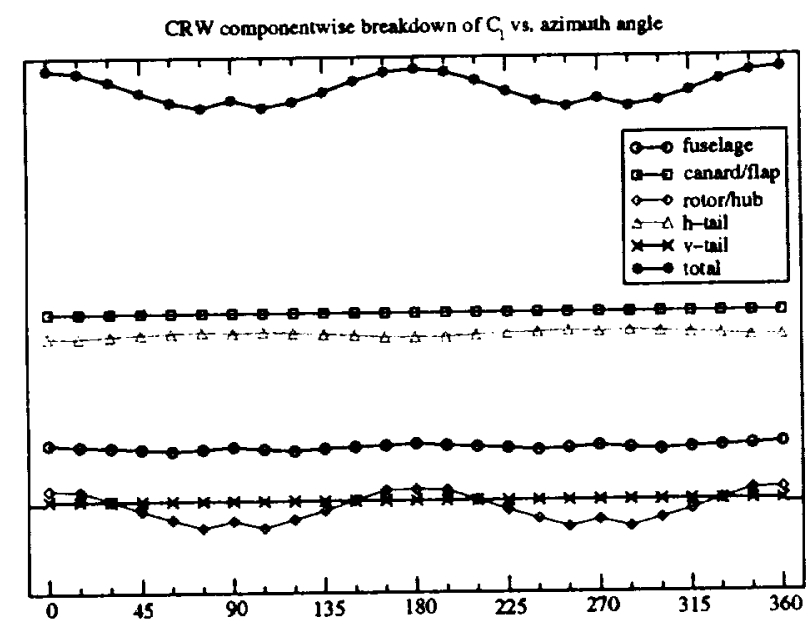

Figure 5: Componentwise breakdown of lift

Having ruled out the downwash from the canard/flap, there are two other factors contributing to these changes in surface pressure under the rotor. One factor is the upwash from the front part of the fuselage. The upwash is a result of the fuselage pushing the flow upward on the front side of the vehicle. When the rotor is close to the fuselage, a large part of the rotor is exposed to this upwash generating forces and moments which diminish as the rotor moves away from the body. Another factor is the suction on the rotor hub fairing assembly. This strong suction is the result of the flow accelerating around the hub. From the surface pressure(Figure 4) we note that the peak suction follows the rotor's azimuthal progress. In other words, the amount of suction seen by the underside of the rotor is larger as the rotor moves away from the body.

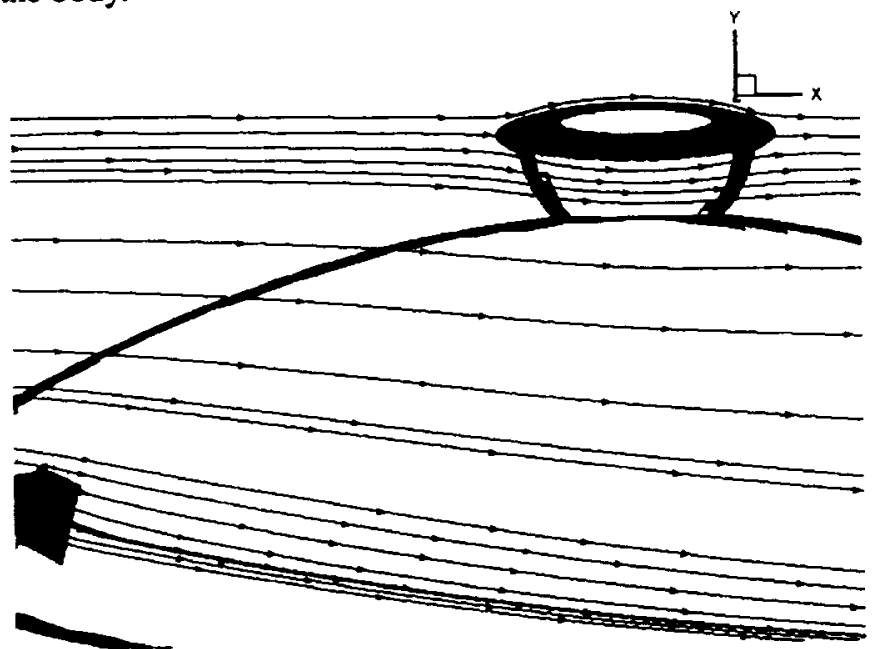

Figure 6: Effect of the downwash from the canard/flap on the rotor
The variations in the suction acting on the horizontal and vertical surfaces around the hub along with the effects of the upwash correlate with the changes in overall vehicle forces and moments. The trends of the flow can be best examined if we look at the variations in forces and moments with respect to the azimuthal position of the rotor and correlate them to the visible changes in pressure on the surface. The plots of the total forces and moments with respect to the azimuthal position of the rotor are presented in figures 7 through 12 . These plots include the curves for the case without the hub, which are discussed in section 3.2 on page 7 . Due to the proprietary nature of this data, the values can not be included on the $y$-axis of figures 7 through 12 .

A plot of the total lift with respect to the azimuthal angle in Figure 7 shows that more lift is being generated when the rotor is in the $0^{\circ}$ position. Upwash from the fuselage is largely responsible for this higher lift. The upwash can clearly be seen in Figure 3 on the front of the fuselage. In Figure 4 , the underside of the rotor at $0^{\circ}$ has higher pressure than when the rotor moves away from the body. This contributes to more lift at $0^{\circ}$ and the lift gradually reduces as the rotor advances since less and less of the rotor is in the upwash field. As the rotor moves away from the body, the suction on the sides of the hub and on the underside of the rotor becomes the dominant effect. As the rotor approaches the $90^{\circ}$ position, the suction under the rotor gets larger. Figure 4 shows the surface pressure from the underside of the rotor so that the hub region is readily visible. We observe that the largest suction on the hub is created when the rotor is in the $90^{\circ}$ position. This suction generates a negative lift on the rotor/hub assembly, which contributes to a lower total lift. Thus, the $90^{\circ}$ case has lower lift than the $0^{\circ}$ case.

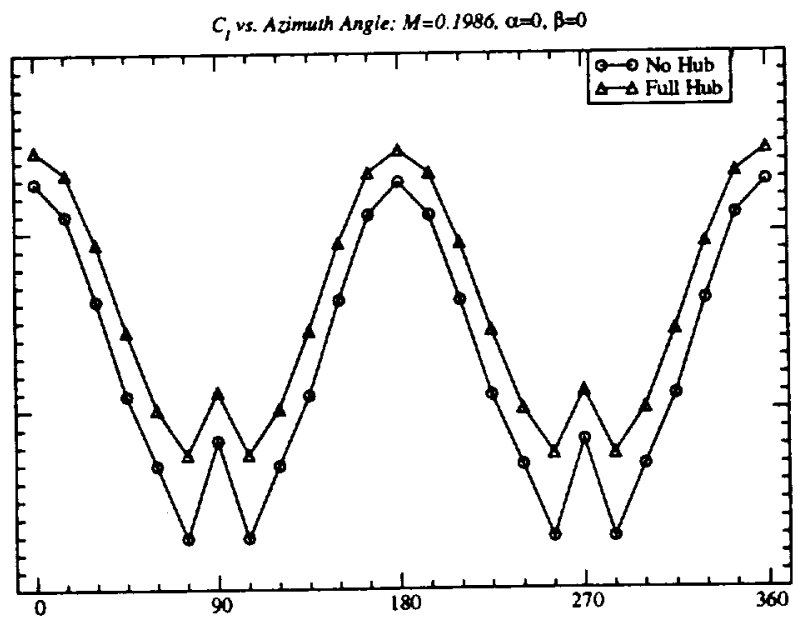

Figure 7: Coefficient of lift

The variation in the drag force, shown in Figure 8 , is much smaller and other effects such as the separated flow behind the hub and the faired-over engine exit may be the primary causes of drag variation. In order for the suction in the hub region to have a direct effect on the drag force, the suction must act on vertical surfaces normal to the flow direction in the vicinity of the hub. Such surfaces do not exist. Thus, the 
movement of the suction in the hub region does not have a large effect on the drag. A possible source of drag is the interference between the rotor and the fuselage. This interference is largest when the rotor is right on top of the fuselage (the $0^{\circ}$ case). The interference drag, therefore is largest at the $0^{\circ}$ rotor position and reduces as the rotor moves away from the body.

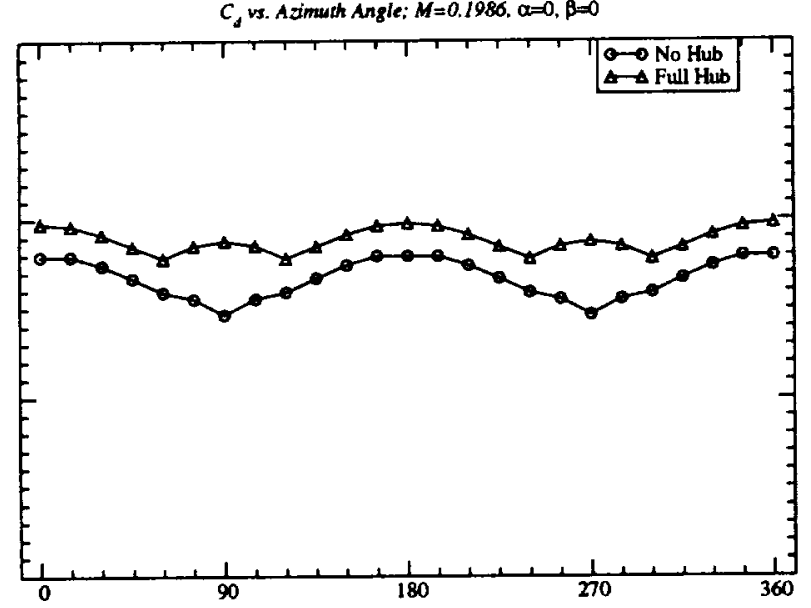

Figure 8: Coefficient of drag

The side force, shown in Figure 9, can also be explained by the tendency of the suction to follow the rotor. In this case, when the rotor is in the $0^{\circ}$ or the $90^{\circ}$ positions, the flow is symmetric and the side force is zero. However, in all other cases, the side force is non zero due to the fact that the suction is higher on one side of the body than the other. This differential suction acts on the vertical surfaces close to the hub, thus generating a side force. The sign of the side force can also be explained by the suction from the hub. If we look at the $45^{\circ}$ case in Figure 4, we see a larger amount of suction on the back rotor as compared to the suction under the front rotor. The result is a positive side force. This is confirmed in Figure 9 where the side force is indeed positive at the $45^{\circ}$ rotor position. The $45^{\circ}$ case should be the same as the $135^{\circ}$ case, but with the opposite effects since the rotor is on the other side of the body. Thus, the value of the side force for the $135^{\circ}$ case should be negative of the $45^{\circ}$ case. The logic carries over to the rest of the cases between $90^{\circ}$ and $180^{\circ}$ and again between $270^{\circ}$ and $360^{\circ}$. The values between $180^{\circ}$ and $270^{\circ}$ are duplicates of the values between $0^{\circ}$ and $90^{\circ}$.

The variation of the moments with respect to the azimuthal angle of the rotor can also be explained in terms of the suction created by the hub. The suction on the hub follows the rotor; thus at a rotor position of $45^{\circ}$, the suction is greater close to where the rotor is. However, due to the oncoming flow, the suction in the front is relieved and so closer to the front facing rotor, there is less suction than the back side. This contributes to a larger downward force on the rearward swept rotor which in turn contributes to a more positive pitching moment. In addition, the upward sloped fuselage induces an upwash on the rotor as evidenced by the higher pressure on the bottom of the front rotor close to the fuselage. This further contributes a positive pitching moment. Because

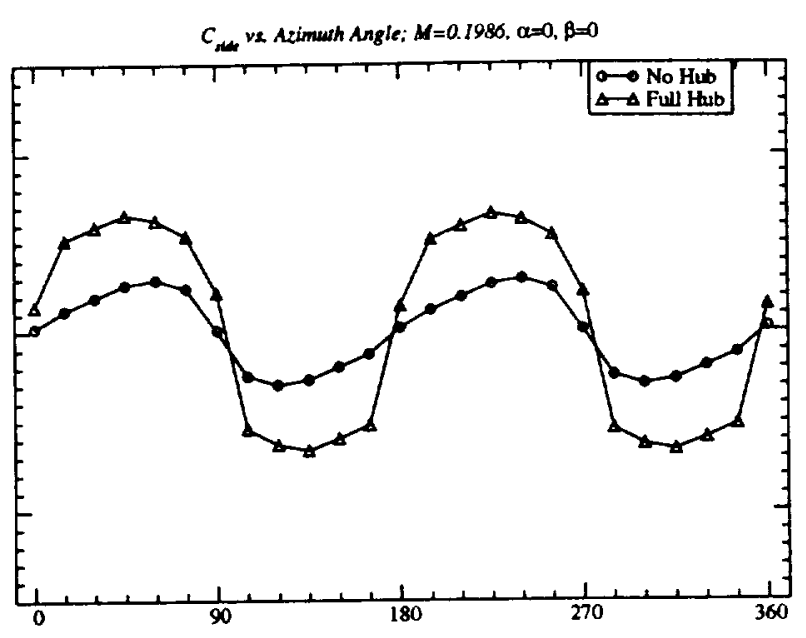

Figure 9: Coefficient of side force

the effect is seen by more of the rotor when the rotor is closer to the body, the moment is higher close to the $0^{\circ}$ position than when most of the rotor is away from the body at the $90^{\circ}$ position. The total variation in pitching moment can be controlled with approximately $2^{\circ}$ of elevon deflection.

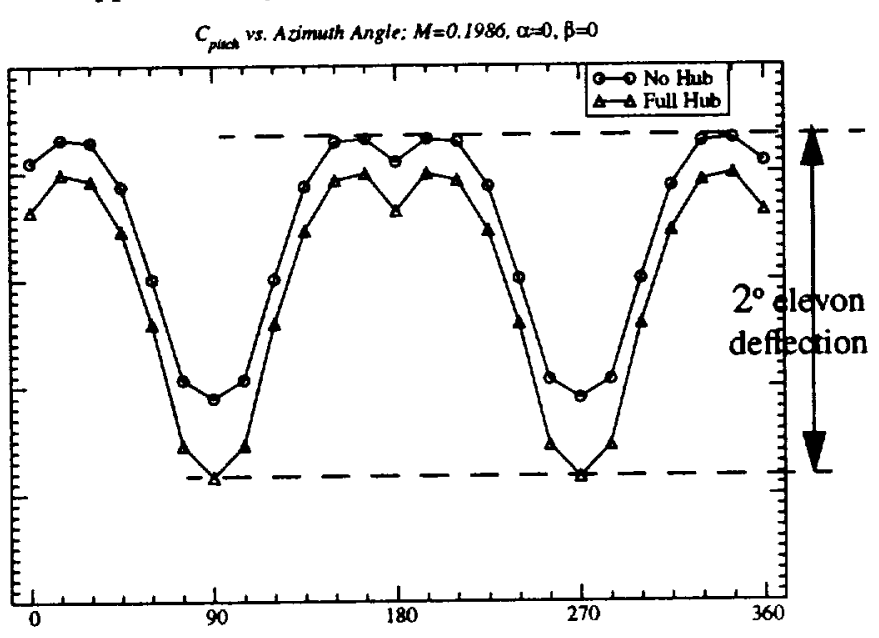

Figure 10: Coefficient of pitching moment

The rolling moment, though much smaller in magnitude than the pitching moment, also depends on the hub suction behavior. Once again, we compare the $0^{\circ}, 45^{\circ}$ and $90^{\circ}$ cases. At 0 and 90 degrees, the rolling moment is zero due to the symmetry of the flow. However, at $45^{\circ}$, the suction has clearly caused the right rotor to produce less lift than the left rotor. For this reason, there is a positive rolling moment produced. The upwash also contributes to a higher lift on the left rotor. This is ascertained from Figure 4 as there is a high pressure region under the left rotor on the side closer to the fuselage. Similarly, a positive rolling moment is produced for the $15^{\circ}$, $30^{\circ}, 60^{\circ}$, and $75^{\circ}$ cases. Like the side force, the symmetry of the flow dictates the sign of the moment at other rotor positions. The total control authority of the elevon is also marked.

A small yawing moment is produced due to the suction force acting differentially on the vertical surfaces close to the hub and its sign can also be explained in terms of the hub suction. 



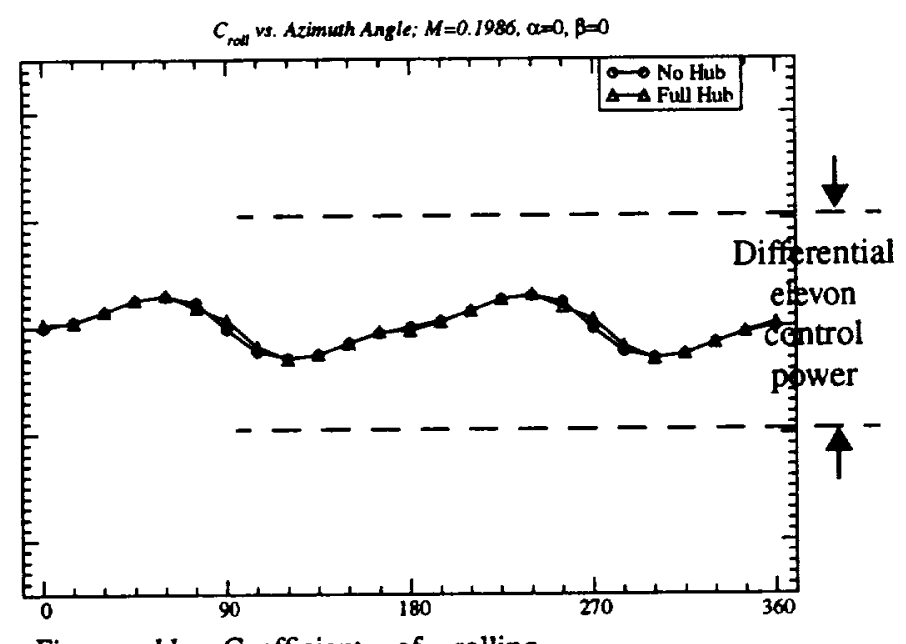

Figure 11: Coefficient of rolling

The yawing moment can be countered with a $3^{\circ}$ deflection of the rudder.

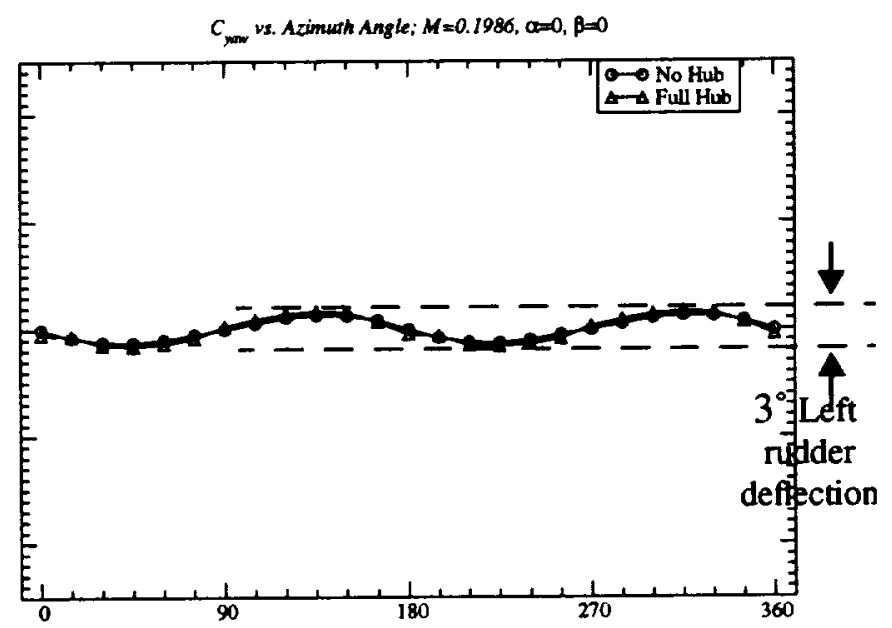

Figure 12: Coefficient of yawing moment

\subsection{Simulations without hub}

To isolate the aerodynamic effects of the hub, a simulation at the same rotor positions as the previous case is conducted without the hub. This is not a realistic configuration since the mast and control arms are not included in the geometry. However, it represents the extreme case and helps understand the effect of the hub on the force and moment trends.

The results are presented in Figure 13 with surface pressure and in figures 7 through 12 with the line plots of the forces and moments with respect to the azimuthal angle. Here also, we look to the surface pressure to explain the trends in the forces and moments. Two reasons to expect differences between the flows over the geometry with the hub and the one without are evident. First, taking the hub away removes the blockage and thus the mechanism for the flow to accelerate around the blockage under the rotor. Second, a channel with top and bottom walls is created with open sides through which flow will accelerate due to the venturi effect. The venturi effect creates a suction that is less severe but more con-

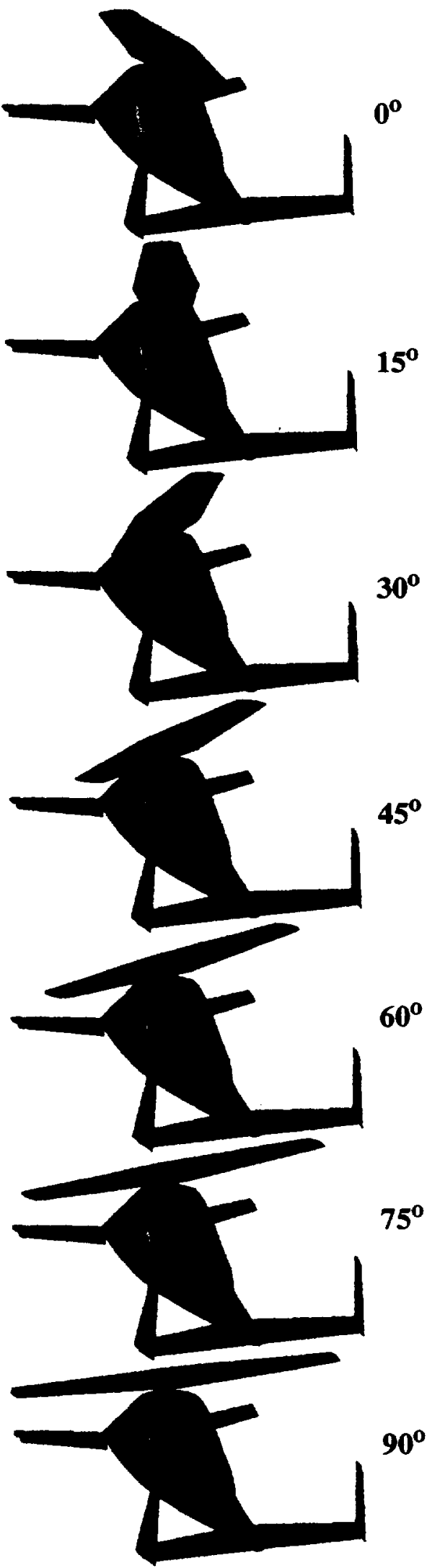

Figure 13: Surface pressure without the hub 

centrated under the center of the rotor than the case with the hub. The trends in forces and moments are very similar to the case with the hub as seen in figures 7 through 12 . We use surface pressure shown in Figure 13 to explain these trends.

The trend in lift is very similar to the case with the hub because just as there was a larger region of low pressure under the rotor for the $90^{\circ}$ case, so it is true for the case without the hub. The surface pressure seen from the underside shows clearly that while the suction due to the accelerated flow is of much smaller magnitude in the $0^{\circ}$ case, it is the dominant feature for the $90^{\circ}$ case. So the rotor generates more negative lift when the rotor tips are away from the body. In addition, the upwash from the fuselage is also present and adds to the higher lift for the cases when the rotor is in the upwash field. We can also explain why the lift is greater for the case with the hub than the case without the hub. The hub has a lifting component in the front and in the rear where the pressure is high due to the fact that the flow is being blocked by the hub. This high pressure contributes to lowering the amount of negative lift contributed by the rotor/hub assembly. The case without the hub has a suction under the rotor, but no high pressure area to counter it. So the overall lift of the case with the hub is larger than the one without the hub.

The changes in drag follow the same trends as the case with the hub for the same reasons. This time, however, the hub is not present which results in less blockage and thus in a lower overall drag.

The side force once again has the same trend, but it is lower than the case with the hub. This is because the flow which spilled over onto the sides of the fuselage from the hub creating a suction region on the vertical walls of the fuselage is no longer present. The non-symmetric effect of the suction under the rotor on the flow on the vertical walls of the fuselage is not as pronounced, resulting in lower side force.

The pitching moment trend is also similar to the case with the hub. Once again we see that the venturi-suction creates a larger suction region on the back rotor and the same arguments we made in the hub case work here. The high pressure due to the upwash from the fuselage on the part of the left rotor close to the fuselage is also contributing positive pitching moment.

The rolling moment is also the victim of the differential suction under the rotor just as the case with the hub is. The upwash field responsible for the higher lift on the left rotor also contributes to the rolling moment as it did for the case with the hub. The actual magnitude is one order smaller than the pitching moment and the difference between the "hubbed" and "hubless" cases is minimal. The upwash is the same in both cases, but the differential suction due to the hub has now been replaced by the differential suction due to the venturi effect.

The yawing moment can also be explained in the same manner and it is also approximately the same as the hub case.

\section{Concluding Remarks}

The vehicle loads on the CRW are evaluated during the conversion from rotor craft to fixed-wing flight using computational tools for inviscid analysis with a Cartesian mesh. The resulting trends in forces and moments are explained in terms of the forces imparted on the body due to the aerodynamic pressure.

It is confirmed that the rotor is indeed unloaded or close to unloaded during conversion. It is also confirmed that the load of the aircraft can be carried by the canard and the horizontal tail. Plots of the variation of the forces and moments with respect to the rotor position are generated and explained in terms of the varying suction in the rotor hub fairing region for the case with the hub and in terms of the varying venturi-suction in the channel between the rotor and the fuselage for the case without the hub. The upwash from the upward slanting fuselage is also responsible in part for the changes in loads with respect to the indexing of the rotor.

The investigation of the results suggests that the case of a hemispherical hub imparts similar loads to the vehicle as the case where the hub has been removed. Though the mechanism for the flow acceleration under the rotor is different in each case, the effective changes in forces and moments are quite similar. The contribution of the upwash field from the front part of the fuselage to the loads on the rotor remains unchanged regardless of the presence of the hub.

The simulations and the investigation of the cause of the variation in the forces and moments suggest that the forces and moments imparted to the geometry may be substantially different when the mast and other hardware are present between the rotor hub and the upper fuselage. This will relieve the suction under the rotor while introducing some blockage in the flow. No changes in the geometry of the hub area can relive the upwash from the forward fuselage. Thus, the effect of the upwash is the limiting case for the load variations experienced by this vehicle. Further study is required to ascertain the changes in loads due to this kind of modification to the geometry.

A non-hemispherical, "more aerodynamic" hub geometry, rather than the complete removal of the hub may also reduce the unsteady loads imparted to the vehicle. However, we also leave this for future investigation.

\section{Acknowledgements}

The authors wish to thank DARPA, Boeing, and Mark Sumich of NASA Ames Research Center for the opportunity to examine the CRW test case and for providing the geometry. We also thank Alex Te for the CAD work he did on this geometry.

\section{References}

[1] McDonnell Douglas Helicopter Systems, Dragonfly advanced technology demonstration, Canard Rotor/ Wing: Fixed-wing wind tunnel test report, Document 98ADRL-B2288-WTTR-009, May 1999 

[2] Aftosmis, M. J., Berger, M. J., Melton, J. E.: "Robust and efficient Cartesian mesh generation for componentbased geometry." AlAA Paper 97-0196, Jan. 1997.

[3] Aftosmis, M. J., Berger, M. J., and Adomovicius, G., "A parallel multilevel method for adaptively refined Cartesian grids with embedded boundaries." AIAA Paper 2000-0808. Jan. 2000.

[4] Aftosmis, M. J., Berger, M. J., and Adomovicius, G. "Parallel multigrid on Cartesian meshes with complex geometry." Proc. of the 8th Intl. Conf. on Parallel CFD. Trondhiem Norway. (To appear Elsivier Press). Jun. 2000.

[5] Melton J. E., Berger, M. J., and Aftosmis, M. J., "3D applications of a Cartesian grid Euler method," ALAA Paper 95-0853-CP, Jul. 1993.

[6] van Leer, Bram, Tai, Chang-Hsien, and Powell, Kenneth G. "Design of Optimally Smoothing Multi-Stage Schemes for the Euler Equations" AIAA Paper 89-1933. 
$-$ 